\title{
ReaR

\section{Competencias profesionales de la función directiva en Unidades de Gestión Clínica}

\section{Paz Martín D.}

\section{Complejo Hospitalario de Toledo}

\section{Resumen}

Los directivos empresariales son cada vez más conscientes que la diferencia entre las organizaciones exitosas a largo plazo y las que no lo son está en las personas.

Como explicó J. Pfeffer "El éxito no procede de tener una buena estrategia, sino de su correcta implantación. Esta capacidad de implantación depende en gran medida de la gente que compone la organización, de cómo son tratados, de sus capacidades y competencias, y de sus esfuerzos por contribuir a la empresa".

A lo largo del siguiente texto se aborda el concepto de competencia que produce comportamientos observables, que es precisamente una de las diferencias con las habilidades. Posteriormente se profundiza en las competencias profesionales de la función directiva comunes a todo tipo de organizaciones y de forma específica aquellas sugeridas para el ámbito sanitario. No se aborda, por entender el autor que se trata de un tema aparte, el concepto de dirección por competencias de las Unidades de Gestión Clínica, que podría definirse como "La evaluación del uso y el nivel de desarrollo de las competencias de una persona (o equipo de la Unidad de Gestión Clínica) para ayudar a la consolidación, adquisición y mejora de comportamientos observables, espontáneos, recurrentes y relevantes para la consecución de resultados en el trabajo profesional"

\section{Introducción}

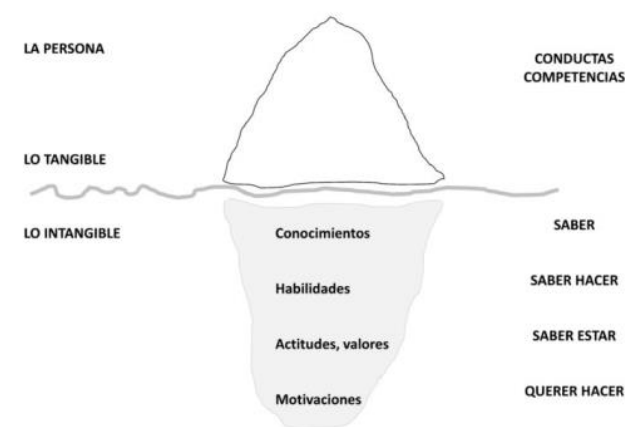

Los directivos empresariales son cada vez más conscientes que la diferencia entre las organizaciones exitosas a largo plazo y las que no lo son está en las personas.

Como explicó J. Pfeffer "El éxito no procede de tener una buena estrategia, sino de su correcta implantación. Esta capacidad de implantación depende en gran medida de la gente que compone la organización, de cómo son tratados, de sus capacidades y competencias, y de sus esfuerzos por contribuir a la empresa".

A lo largo del siguiente texto se aborda el concepto de competencia que produce comportamientos observables, que es precisamente una de las diferencias con las habilidades. Posteriormente se profundiza en las competencias profesionales de la función directiva comunes a todo tipo de organizaciones y de forma específica aquellas sugeridas para el ámbito sanitario. No se aborda, por entender el autor que se trata de un tema aparte, el concepto de dirección por competencias de las Unidades de Gestión Clínica, que 
podría definirse como "La evaluación del uso y el nivel de desarrollo de las competencias de una persona (o equipo de la Unidad de Gestión Clínica) para ayudar a la consolidación, adquisición y mejora de comportamientos observables, espontáneos, recurrentes y relevantes para la consecución de resultados en el trabajo profesional"

\section{Función directiva}

La función directiva ha sido definida como el proceso, que comprende funciones $\mathrm{y}$ actividades sociales $\mathrm{y}$ técnicas, que ocurre en las organizaciones con el propósito de alcanzar objetivos predeterminados a través de recursos humanos y materiales (1).

Queda implícito en la definición anterior, que los directivos trabajan con otras personas, llevando a cabo actividades técnicas e interpersonales para alcanzar los objetivos de la organización.

Pérez López en su modelo antropológico de la empresa propone que la función directiva consiste en diseñar estrategias que produzcan valor económico, desarrollando las capacidades de sus empleados y uniéndolos con la visión de la empresa (2).

\section{Dimensión estratégica e intratégica de la función directiva}

Como dice Covey, la efectividad de la empresa reside en el equilibrio $\mathrm{P} / \mathrm{CP}$; donde $\mathrm{P}$ es la producción de los resultados deseados y CP la capacidad de producción (3). De esta forma dentro de la función directiva se incluiría una:

- Dimensión estratégica orientada a los beneficios económicos (o de salud como en nuestro medio).
- Dimensión intratégica dirigida al desarrollo de los empleados y al incremento de su compromiso con la organización (4).

Si una organización sanitaria no invierte continuamente para mejorar su $\mathrm{CP}$ limitará severamente sus opciones a medio plazo. El director de la Unidad de Gestión Clínica (UGC) tiene la corresponsabilidad de conseguir el máximo desarrollo profesional de cada persona implicada en la misma.

\section{Unidad de Gestión Clínica}

La Gestión Clínica es todo proceso de rediseño cuyo objetivo es incorporar al profesional sanitario en la gestión de los recursos utilizados en su propia práctica. Esto lleva a la utilización adecuada de los recursos para la mejor atención de los pacientes, desde la responsabilidad del profesional sanitario.

La UGC es una estructura organizativa donde se desarrolla la gestión por procesos en el ámbito clínico.

La Gestión Clínica se caracteriza por (5):

- Autonomía de gestión y recursos propios. Corresponsabilidad del profesional médico en la gestión de los recursos

- Grupo de pacientes caracterizado que optarán por los servicios.

- Agrupación de profesionales de diferentes servicios/estamentos con el objetivo de alcanzar la máxima excelencia y eficiencia asistencial.

- Educación para la salud e implicación del paciente en las decisiones clínicas

- Continuidad asistencial

- Práctica clínica de calidad; definición de procesos, liderazgo 
clínico y elaboración de planes terapéuticos individualizados

- Evaluación de procesos, pero sobre todo de resultados clínicos, además de los costes necesarios para su obtención

\section{Función directiva en las Unidades de Gestión Clínica}

En las UGC se dan dos tipos de actividades:

- Programadas: o de mera ejecución.

- Directivas: son aquellas que suplen todo lo que les falta a las actividades programadas para que los problemas sean resueltos de hecho.

Los directores de las UGC deben desarrollar funciones en 3 áreas de actividad

- Actividades estratégicas: formulación de los objetivos de la UGC, en línea con los objetivos organizacionales.

- Actividades ejecutivas: planificación, organización, dotación y monitorización del desempeño de los individuos del grupo y aplicación de determinadas acciones correctivas. El objetivo último de este grupo de actividades es alcanzar las metas de la UGC.

- Actividades de liderazgo: motivación del equipo para que desarrollen aquellas actividades.

\section{Competencias}

De cara a desarrollar las anteriores funciones, el director de UGC necesita de una serie de capacidades o competencias claves. Estas se encontrarían dentro de tres dimensiones distintas del directivo:

- Su cualidad de estratega

- Su cualidad de ejecutivo

- Su cualidad de líder

Las diferentes organizaciones pueden necesitar más un talento que otro en momentos determinados y según las circunstancias. Por este motivo no sería pertinente intentar describir al director de UGC ideal, sin embargo, trataremos de desarrollar aquellas competencias directivas comunes a todos los directivos de organizaciones sanitarias.

Según la Real Academia de la Lengua Española una de las acepciones de competencia sería: Pericia, aptitud o idoneidad para hacer algo o intervenir en un asunto determinado.

El termino competencia en el contexto del management fue empleado por primera vez por David Mc Clelland en 1973 en su artículo Testing for competence rather tan intelligence (6). El motivo de su aparición fue la búsqueda de aquellas características que mejor predijeran la idoneidad de un profesional en su puesto de trabajo.

Pereda y Berrocal definieron competencia como "Un conjunto de comportamientos observables que están causalmente relacionados con un desempeño concreto $\mathrm{y}$ en una organización concreta" (7). Las competencias, por tanto, no implican sólo una serie de conocimientos específicos, sino el desarrollo de la capacidad de utilizarlos como herramientas para afrontar situaciones problemáticas. 


\section{Competencias Directivas}

\section{Modelo del Iceberg.}

Las competencias están formadas por (Fig. 1):

- Conocimientos y percepciones: representación interna de la realidad (SABER).

- Habilidades, destrezas, estrategias y procedimientos: ejecución práctica (SABER HACER).

- Actitudes y valores: disposición de ánimo y normas que rigen nuestra conducta y que han sido elegidas libre y conscientemente (SABER ESTAR).

- Motivaciones: HACER).

(QUERER

Las competencias dan lugar a comportamientos observables que son relevantes para el buen desarrollo de la tarea. En tanto son comportamientos observables se encontrarían en el nivel más externo que cada persona muestra al desempeñar una función en una organización.

La dirección por competencias no pondría el foco en los resultados de la tarea, sino en aquellas características de la persona que propician que se produzcan $\mathrm{y}$ en la forma que se consiguen.

Es importante distinguir que existen dos tipos fundamentales de competencias: las técnicas o de puesto y las directivas (3).

\section{Competencias Técnicas}

Aquellas que requiere un trabajador en un puesto de trabajo determinado.
Son aquellos comportamientos observables y habituales que posibilitan el éxito de una persona en su función directiva (4).

Dentro de este grupo se podrían subdividir en:

- Competencias estratégicas: necesarias para obtener buenos resultados.

- Visión de negocio: capacidad de reconocer los diferentes peligros $\mathrm{y}$ aprovechar las fortalezas que afectan a la eficacia del negocio.

- Gestión de recursos: se trata del empleo de los recursos materiales y humanos de la mejor forma y a menor coste para alcanzar los objetivos deseados.

- Resolución de problemas

- Orientación al cliente: capacidad de alcanzar la satisfacción del cliente e incorporar sus sugerencias en la mejora de los procesos.

- Competencias intratégicas: necesarias para desarrollar al equipo e incrementar su compromiso

- Empatía: es la capacidad de identificarse con alguien y compartir sus sentimientos.

- Delegación:

- Coaching

- Comunicación

- Competencias de eficacia personal: un buen directivo debe 
saber dirigirse a sí mismo. Nadie da lo que no tiene. El término "Inteligencia Emocional" fue acuñado por Daniel Goleman para designar una competencia indispensable en el directivo como es la capacidad de dirigir sus emociones (8).

Las competencias de eficacia personal fueron definidas por Covey como aquellos hábitos que facilitan una relación eficaz con el entorno (3).

- Victoria privada: tiene como objetivo pasar de ser dependientes del entorno a ser independientes.

- Hábito 1: Ser proactivo

- Hábito 2: Comenzar con un fin en mente

- Hábito 3: Poner primero lo primero

- Victoria pública: estos hábitos permiten dar el salto a la interdependencia

- Hábito 4: Pensar ganarganar

- Hábito 5: Buscar primero entender, luego ser entendido

- Hábito 6: Sinergizar

- Renovación

- Hábito 7: Afilar la sierra.

A pesar de que muchas de las competencias directivas son comunes a los diferentes tipos de industrias, lo cierto es que cada organización debería de decidir qué competencias prefiere desarrollar entre sus directivos. Recientemente Carson Dye y Andrew Garman (9) han publicado las 16 competencias que deberían presentar los directores de las organizaciones sanitarias y que clasificaron en 4 aspectos claves:

\section{Autoconsciencia bien cultivada}

\section{Visión convincente \\ 3. Un camino real con la gente \\ 4. Ejecución magistral}

\section{Autoconsciencia bien cultivada}

\section{Competencia $1-$ Liderar con convicción.}

El director de UGC debe identificar sus valores y creencias, y permitir que guíen la toma de decisiones, incluso si esto conduce a decisiones impopulares. Esta convicción debe ser demostrada a través del trabajo que realiza. El director excelente debería, de forma simultánea, reconocer que sus convicciones personales no son universales. Cada decisión está impregnada de una dimensión ética y los líderes altamente efectivos deberían de mostrar una alta integridad y ética. La falta de integridad podría llegar a socavar casi cualquier otro esfuerzo para crear relaciones de confianza con el equipo.

\section{Competencia 2 - Uso de la inteligencia emocional.}

Es importante que el director de UGC reconozca sus fortalezas y debilidades y maneje sus emociones. La efectividad a largo plazo dependerá de las relaciones que se establezcan en la UGC que en última instancia estarán determinadas por la capacidad del grupo, y especialmente del líder, de gestionar emociones.

Los directores de UGC deben mantener un equilibrio entre mantener unas relaciones de confianza, familiaridad y confort con el grupo, y a la vez cierta distancia emocional que les permita valorar con objetividad cada escenario teniendo en cuenta los mejores intereses de la organización.

El uso de la inteligencia emocional es la base de una delegación efectiva que es 
la capacidad de conseguir que los colaboradores del equipo dispongan de la información y los recursos necesarios para tomar decisiones y lograr sus objetivos

\section{Competencia 3 - Ganar confianza y lealtad}

El líder de la UGC debe ser una persona directa y veraz, dispuesta a admitir errores, que demuestra con sus acciones los compromisos adquiridos y que presta atención a las preocupaciones de los demás. Desarrollar relaciones de confianza entre el director y el equipo es extraordinariamente relevante para el desarrollo de un liderazgo efectivo. La confianza mantiene unido al grupo y a la organización.

Para ganar la confianza del equipo, el director de la UGC debe:

- Ser accesible: debe ser abierto, franco y compartir la información libremente.

- Fomentar la apertura: la confianza se desarrolla a lo largo del tiempo, no hay soluciones rápidas. Se debe cultivar un ambiente de seguridad psicológica en el que cada individuo sienta que puede hablar honestamente sin ser juzgados. El director debe buscar primero escuchar con empatía, comprender realmente, durante el tiempo que sea necesario antes de ser entendido.

- Ser auténtico: la confianza la ganan los líderes que cumplen sus promesas (ratio hacer/decir). Los compromisos con los demás, pero también con nosotros mismos, y la integridad con que los mantenemos son la esencia de la proactividad.

- Dar ejemplo: un director de UGC no debería pedir nada que no estuviera dispuesto a realizar él mismo.

- Transformar la confianza en lealtad.

\section{Competencia 4 - Dar energía al equipo.}

El director de la UGC debe dar ejemplo personal de ética profesional $y$ motivación, debe hablar y actuar de forma entusiasta y optimista hacia el futuro, disfrutar con los nuevos retos, y mostrar energía y pasión para la consecución de objetivos. Así mismo, es importante que ayude a su equipo a reconocer la importancia, he incluso a disfrutar, de su trabajo. Los líderes que estimulan a su personal deben:

- Comprender los objetivos y las prioridades individuales.

- Celebrar y compartir los éxitos.

- Tener sentido del humor: que no es en ningún caso incompatible con mantener la necesaria seriedad en el desarrollo de las funciones.

\section{Visión convincente}

\section{Competencia 5 - Ser Visionario.}

El líder de la UGC debe anticipar aquellos cambios a gran escala o a nivel local que podrían llegar a afectar a la organización. La anticipación de los cambios en base a una adecuada interpretación de las tendencias actuales conferiría al director una mayor capacidad para poner en marcha estrategias que permitan a la UGC adaptarse a ellos.

La visión de negocio sería la capacidad de reconocer los peligros y aprovechar las oportunidades y las fuerzas externas que repercuten en la competitividad y efectividad del negocio. 
Sin duda alguna, los mejores líderes son los que son capaces de trascender el día a día y mirar hacia el futuro.

\section{Competencia 6 - Comunicación de la visión.}

El director de UGC debe ayudar a las partes interesadas a comprender el estado futuro deseado y comunicar su papel para alcanzar ese estado. Comunicar la visión durante cualquier proceso de cambio es esencial para su éxito.

Muchos líderes a menudo subestiman la importancia de una comunicación efectiva lo que puede acabar desmotivando al equipo que podría dejar de lado los cambios sugeridos y volver a sus antiguos hábitos.

Los directores efectivos no sólo comunican sobre aspectos operativos del día a día, sino que trasladan su visión al equipo a través de:

\section{- Comunicación clara.}

- Comunicación amplia: en el sentido que llega a todos los individuos que debe llegar y a través de un número suficiente de canales, formales e informales, que permitan que el mensaje se transmita de forma efectiva. Es un error frecuente que el director de la UGC envíe un mensaje a través de correo electrónico y considere que la comunicación está cerrada. El mensaje no debe ser sólo leído o escuchado sino también discutido por el equipo.

\section{Un camino real con la gente.}

\footnotetext{
Competencia 7 - Escuchar con empatía.
}

La mayor parte de las personas no escuchan con la intención de comprender, sino de contestar. Según Covey cuando otra habla por lo general se escucha en cuatro niveles (3):

\section{Ignorándola}

- Fingimos que la escuchamos

- Escucha de forma selectiva

- Escucha con atención

El propio Covey propone un quinto nivel de escucha que correspondería a Escuchar con Empatía. En este tipo de escucha se busca primero entender profundamente al que habla y después ser entendido. No es sólo ser accesible al otro $y$ de mente abierta, sino comprender su paradigma, comprender lo que siente.

Escuchar con empatía es una de las formas más eficaces que tiene un director de una UGC de aumentar la confianza que tiene su equipo en él y de entender mejor los objetivos, las prioridades y las perspectivas de su equipo.

\section{Competencia 8 - Dar feedback.}

Feedback podría ser definido como la realización de comentarios sobre el desempeño. El líder debe establecer expectativas claras y ofrecer críticas y elogios cuando sea apropiado. Existen una serie de recursos que mejoran el feedback

- Realizar el comentario tan pronto como sea posible al comportamiento al que va dirigido.

- Describir objetivamente lo observado. 
- Sugerir un área específica de mejora.

- Terminar con una nota positiva.

- Realizar el comentario en un entorno adecuado de seguridad psicológica que minimice la posibilidad de que se den conductas excesivamente defensivas y mejore el efecto deseado del comentario.

\section{Competencia 9 - Mentoring.}

Comprende todas las acciones que los líderes (o mentores) llevan a cabo para desarrollar las capacidades de su personal más acordes con su potencial para satisfacer los requerimientos globales de la organización. Para esto es indispensable:

- conocer las aspiraciones individuales de cada miembro del equipo.

- exige un compromiso a largo plazo con esta tarea.

- participación activa del líder en el desarrollo de las habilidades de su equipo.

- comprender que, aunque un desarrollo profesional de los integrantes del equipo pueda facilitar su empleabilidad en otras organizaciones, los beneficios de esta tarea superan claramente estos inconvenientes.

\section{Competencia 10 - Desarrollar equipos de alto rendimiento.}

En UGC los miembros del equipo tienen las siguientes características:

- tienen objetivos comunes.
- el éxito en la consecución de estos objetivos depende de la interdependencia.

En el desarrollo de equipos de alto rendimiento existen 5 actividades críticas.

1. Conseguir las mejores personas para los roles de equipo.

- Desarrollar su orientación hacia una visión común y objetivos colectivos.

- Desarrollar la confianza entre los miembros del equipo.

- Desarrollar la cohesión entre los miembros del equipo.

- Aumentando la frecuencia de interacciones tanto en el trabajo como fuera del trabajo.

- Facilitando que el grupo pueda discutir sobre los objetivos a alcanzar y cómo deben ser alcanzados.

- Estableciendo relaciones ganar-ganar en el seno del grupo. Todos los miembros de la UGC deben sentirse ganadores. La tercera vía es posible.

- Ayudar a los miembros del equipo a trabajar de manera productiva a través de los inevitables conflictos que vienen con la interacción grupal. El conflicto siempre, siempre va a estar presente $y$ hay que aprender a gestionarlo:

- asegurando que haya equidad en la asignación de recursos entre los miembros del equipo.

- minimizando el crecimiento de subgrupos de influencia, 
- manteniendo las reacciones personales fuera de los límites del conflicto,

- asegurando que haya una mínima ambigüedad de roles entre los miembros del equipo,

- asegurando que las discusiones en equipo tengan lugar dentro de los límites del equipo.

\section{Ejecución magistral}

\section{Competencia 11- Generación de poder informal.}

Generar poder informal significa que el líder de la UGC tiene la capacidad de influir sobre otros sin tener que recurrir a su autoridad formal. Para ello es imprescindible entender los diferentes roles de poder e influencia en las organizaciones, al margen del organigrama formal, y desarrollar argumentos convincentes o puntos de vista basados en el conocimiento de las prioridades de otros para influenciarlos. El líder debe desarrollar y mantener redes útiles de influencia hacia arriba, hacia abajo y hacia los lados de la organización.

Según French y Raven existen 6 tipos fundamentales de poderes (10):

1. Formal (Legítimo): proviene de la posición que ocupa una persona (ej. tienes que hacer lo que te pida porque yo soy el jefe).

- Recompensa. Este poder se deriva de la capacidad de proporcionar beneficios (ej. compensación económica, ascenso).

- Penalidad (Coercitivo). Este poder nace de la capacidad de sancionar a los miembros del equipo.

- Experto. Este poder proviene de un conocimiento o una experiencia especial.

- Referente. El poder de referencia proviene de la admiración o la alta consideración que se tiene por alguien.

- Información: derivado de la posesión de determinada información o datos.

Sin duda alguna las mejores formas de poder para el liderazgo efectivo de una UGC son el poder experto y el de recompensa (pensar ganar-ganar).

\section{Competencia 12 -Crear un verdadero consenso.}

El consenso puede definirse como acuerdo general. La construcción de consenso implica el uso de decisiones grupales y otras técnicas para facilitar alcanzar un umbral crítico de acuerdo general.

La mayor parte de las decisiones que debe tomar un líder no produce resultados iguales para todos los afectados. Crear consenso implica desarrollar los niveles de apoyo necesarios para hacer avanzar las iniciativas sin hacer que algunas personas se sientan abandonadas mientras otras resulten victoriosas (relación perder-ganar).

Estas soluciones (perder-ganar o ganarperder) a medio plazo son inestables originan conflictos que el director de la UGC deberá afrontar.

Existen diferentes estilos de decisión. Cardona (4), basándose en la matriz 
importante/urgente de Covey expuso la siguiente propuesta (3):

El eje vertical reflejaría la importancia de la decisión, mientras que en el horizontal correspondería a la urgencia de la misma. Siguiendo esta matriz importancia/urgencia se puede determinar cuál es el estilo adecuado para tomar la decisión en cada momento.

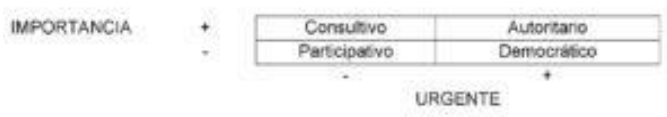

Tabla 1. Matriz de estilo de decisión.

Estilo participativo: es el más adecuado para aquellas decisiones poco urgentes y poco importantes (delegables). Es en estas, que como veremos más adelante son las más frecuentes, en las que el director de la UGC debe alcanzar los consensos.

Estilo democrático: adecuado para decisiones que son urgentes, pero poco importantes (ej. ¿Dónde celebramos la despedida de los residentes de último año?)

Estilo consultivo: decisiones que competen exclusivamente al director de la UGC pero no son urgentes. En estos casos, lo razonable es que el director solicite opiniones del resto del equipo antes de tomar la decisión.

Estilo autoritario: necesario en aquellas decisiones que competen al director de la UGC y que son muy urgentes.

El propio Cardona propone una distribución de estos estilos siguiendo el principio de Pareto. Recordemos que el Principio de Pareto establece que el 80 por ciento de los problemas se deben al 20 por ciento de las causas raíz.
Siguiendo esta regla del $80 / 20$ el $20 \%$ de las decisiones no serían delegables frente a un $80 \%$ que si lo serian, y un $20 \%$ serían urgentes frente a un $80 \%$ que serían demorables.

Incorporando los datos anteriores en la matriz quedaría de la siguiente forma:

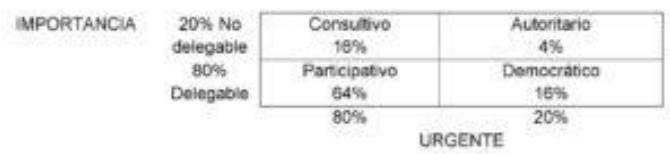

Tabla 2. Porcentajes en la Matriz de estilos de decisión.

Cuando se requiere el consenso se debe asegurar que los siguientes elementos estén bien atendidos:

- Equilibrio: No todas las decisiones deben tomarse por unanimidad, pero el director de la UGC debe buscar un consenso suficientemente bueno para cada decisión determinada. Por tanto, el nivel de consenso variará en cada circunstancia.

- Eficiencia: el alcance de determinados consensos puede requerir de un tiempo y dedicación notable por parte del líder. Hay que tratar que este proceso se realice de la forma más eficientemente posible.

- Técnica: es importante que el líder desarrolle habilidades que le permitan abordar de forma efectiva a los diferentes profesionales del equipo

\section{Competencia 13 - Toma de decisiones efectiva.}

El director de la UGC debe tomar decisiones basadas en una combinación de hechos, objetivos, ética, alternativas $y$ juicios. La toma de decisiones efectiva puede apoyarse en herramientas de decisión (ej. análisis de costobeneficio, árboles de decisión). 
Es importante destacar que hay cierta tendencia a pensar que la toma de decisiones es una cualidad inmutable, innata al líder. En realidad, el proceso de toma de decisiones no sólo se basa en la inteligencia y experiencia del que las toma, sino que se ve influido por una serie de habilidades aprendibles $y$ refinables, como son:

- Tener sentido del tiempo: es importante que el director de la UGC identifique aquellas circunstancias que requieren una acción inmediata y aquellas que se beneficiarán de mayor tiempo, análisis y otros puntos de vista.

- Enfoque óptimo en la toma de decisiones: el líder debe "decidir cómo decidir" y para ello puede hacerse varias preguntas:

- ¿Quién se verá afectado por el resultado de la decisión?

- ¿Qué rol deberían tener determinados individuos (grupos) en el proceso de toma de decisiones?

- ¿Cuáles son los aspectos claves que se deben tener en cuenta para poder tomar una decisión específica?

- Cantidad de atención prestada en el análisis de la decisión: en la recopilación de información, identificación de las diferentes opciones y la valoración de la relación riesgo/beneficio de cada una de ellas. Como todo este proceso consume tiempo $\mathrm{y}$ recursos a menudo es preferible un abordaje suficientemente bueno del problema que profundizar excesiva e innecesariamente en el mismo. De hecho, la capacidad para tomar decisiones sin estar completamente seguro de disponer de toda la información es una habilidad necesaria en la gestión de organizaciones sanitarias.

- Poseer buen juicio: hay dos aspectos esenciales que caracterizan a aquellos que toman mejores decisiones:

- 1) exploran un abanico más amplio de opciones y

- 2) tienen una mejor capacidad para explicarlas.

- Actuar con consistencia e integridad: esto aumenta la confianza del equipo en el director de la unidad que no se verá obligado a justificar cada una de sus decisiones porque de forma habitual las toma de una forma metódica y justa.

\section{Competencia 14 - Gestión orientada a los objetivos.}

Moviliza al equipo hacia la obtención de altos estándares de calidad y hacia un mayor compromiso. Monitoriza y comunica la evolución de los diferentes proyectos y aplica las medidas correctivas necesarias para alcanzar las metas.

La gestión orientada a objetivos es a menudo lo que diferencia los equipos que realizan esfuerzos adicionales de aquellos que se limitan a cumplir con sus tareas asistenciales.

Cuando los directores de UGC orientan al equipo por objetivos:

- Buscan proactivamente oportunidades de mejora de procesos: esta búsqueda parece reflejar más una orientación general de mejora continua que 
una herramienta que se debe utilizar para solucionar problemas identificados.

- Mantienen el foco en la mejora de procesos: no sólo detectan oportunidades, sino que se mantienen y mantienen al equipo centrados en esa tarea.

- Son organizados: realizan una monitorización adecuada de los hitos que se deben alcanzar.

- Tienen energía ilimitada: más allá de ser productivos, los líderes excepcionales, a menudo son descritos como imparables. En su día a día tienden a ver las barreras como temporales y se mueven alrededor de ellas de la forma más eficientemente posible.

\section{Competencia 15 - Estimular la creatividad.}

El líder de la UGC debe estar abierto a nuevas ideas y formas de abordar el trabajo o los desafíos. Frecuentemente identifica futuras tendencias y aprende de las que presentan otras industrias.

Creatividad podría ser definida como el uso de enfoques innovadores en la resolución de problemas y la toma de decisiones. El establecimiento de un entorno que permita estimular la creatividad de las personas con las que trabajan, es una habilidad crítica de los directores innovadores.

Estimular la creatividad implica:

- Tener una actitud positiva hacia los retos: de esta forma dejan de ser un inconveniente del que hay que deshacerse rápidamente para ser una oportunidad de crecimiento.
- Fomentar diferentes perspectivas: los enfoques creativos requieren comprender las perspectivas de los interesados para poder considerarlas también.

- Impulsar el desarrollo ideas creativas: dentro del equipo mediante la creación de un ambiente de seguridad psicológica en la que ningún miembro pueda sentirse vulnerable.

- Construir soluciones creativas: sintetizando las diferentes ideas expuestas en un todo coherente.

\section{Competencia 16 - Cultivando la adaptabilidad.}

El líder que cultiva la adaptabilidad interpreta rápidamente la esencia de los problemas y aporta claridad a las situaciones ambiguas.

Las prioridades son cambiantes y un director de UGC debe interpretar fácilmente los nuevos escenarios, sus implicaciones, y tener capacidad de redirigir el rumbo según sea necesario.

La adaptabilidad implica el dominio de tres habilidades fundamentales:

1. leer el ambiente: capacidad de apreciar los aspectos más importantes de una situación determinada.

2. sopesar los cursos de acción apropiados: determinar qué enfoque funcionará

3. responder con un estilo de liderazgo apropiado (véase anteriormente French y Raven existen 6 tipos fundamentales de poderes). 


\section{Conclusión}

Un escenario sanitario en constante evolución requiere nuevos enfoques $\mathrm{y}$ perspectivas. La presencia de líderes con competencias directivas desarrolladas al frente de las UGC se convertirá en una herramienta necesaria para adaptarse a los nuevos desafíos sanitarios.

\section{Bibliografía}

1.- Longest BB, Rakich JS, Darr K 2000. Managing health services organizations ans systems. Baltimore: Health Professions Press (Sciepub)

2.- Pérez López JA. Liderazgo y ética en la dirección de empresas. Deusto. Bilbao 1998.

3.- Covey ST. Los 7 hábitos de la gente altamente efectiva. La revolución ética en la vida cotidiana y en la empresa. Ed Paidós 2015.

4.- Álvarez De Mon. Paradigmas del liderazgo. Claves de la dirección de personas. 2 Edición. ED McGraw-Hill de Management 2001.

5.- Porter, ME, and Thomas H. Lee. "The Strategy That Will Fix Health Care." Harvard Business Review 912013; 91: 50-70. (HTML)

6.- Mc Clelland DC Testing for competence rather than intelligence. American Psychologist 1973;28(1):1-4. (

7.- Pereda S, Berrocal F. Gestión de Recursos Humanos por Competencias. Madrid: Centro de Estudios Ramón Areces. 1999.

8.- Goleman D: La inteligencia emocional. Ed Paidos 1994

9.- Dye CF, Garman AN Exceptional leadership: 16 critical competencies for health executives. Health Administration Press 2006
10.- Longest BB, Rakich JS, Darr K 2000. Managing health services organizations ans systems. Baltimore: Health Professions Press ( $\underline{\text { Sciepub}})$

11.- Pérez López JA. Liderazgo y ética en la dirección de empresas. Deusto. Bilbao 1998.

12.- Covey ST. Los 7 hábitos de la gente altamente efectiva. La revolución ética en la vida cotidiana y en la empresa. Ed Paidós 2015.

13.- Álvarez De Mon. Paradigmas del liderazgo. Claves de la dirección de personas. 2 Edición. ED McGraw-Hill de Management 2001.

14.- Porter, ME, and Thomas H. Lee. "The Strategy That Will Fix Health Care." Harvard Business Review 912013; 91: 50-70. (䶊)

15.- Mc Clelland DC Testing for competence rather than intelligence. American Psychologist 1973;28(1):1-4. (

16.- Pereda S, Berrocal F. Gestión de Recursos Humanos por Competencias. Madrid: Centro de Estudios Ramón Areces. 1999.

17.- Goleman D: La inteligencia emocional. Ed Paidos 1994

18.- Dye CF, Garman AN Exceptional leadership: 16 critical competencies for health executives. Health Administration Press 2006

Correspondencia al autor

Daniel Paz Martín

dpaz@anestesiar.org

MD PhD EDAIC EDIC PMP Sección Cuidados Intensivos de la SEDAR

Jefe de Sección Anestesiología y Reanimación. Complejo Hospitalario de Toledo

Presidente de AnestesiaR

Aceptado para el blog en octubre de 2019. 